\title{
Controle Preditivo Robusto com Restrições aplicadas ao Diagrama de Nyquist ${ }^{\star}$
}

\author{
Alequine Batista de Lima* \\ Péricles Rezende Barros, George Acioli Júnior** \\ * Mestrando do Programa de Pós Graduação em Engenharia Elétrica, \\ Departamento de Engenharia Elétrica, Universidade Federal de \\ Campina Grande, PB, (e-mail: alequine.lima@ee.ufcg.edu.br). \\ ** Departamento de Engenharia Elétrica, Universidade Federal de \\ Campina Grande, PB, (e-mail: prbarros@dee.ufcg.edu.br, \\ georgeacioli@dee.ufcg.edu.br
}

\begin{abstract}
In this work, the authors aim to present a predictive control design technique that is robust, using constraints associated with the robustness of the system while in closed loop. In order to solve an optimization problem, genetic algorithm is used. This constraints are imposed at the Nyquist diagram. A didactic environment is used to validate the technique, consisted of a arduino UNO, MATLAB and Simulink®.

Resumo: Neste trabalho, os autores objetivam apresentar uma técnica de projeto de controlador preditivo que seja robusto, mediante a implementação de restrições associadas à robustez do sistema quando em malha fechada. Para realizar um problema de otimização, algoritmo genético é utilizado. Estas restrições são impostas no diagrama de Nyquist. Um ambiente didático é utilizado para validar a técnica, consistindo de arduino UNO, MATLAB e Simulink@.
\end{abstract}

Keywords: Didactic, Predictive Control, Robustness, Identification, Nyquist, Optimization, Genetic Algorithm.

Palavras-chaves: Didático, Controle Preditivo, Robustez, Identificação, Nyquist, Otimização, Algoritmo Genético.

\section{INTRODUÇÃO}

Nas últimas cinco décadas, se deu um desenvolvimento rápido da tecnologia e da teoria dos controladores preditivos baseados em modelos $(M P C)$. Desde sua inserção no contexto industrial na década de 1970, a partir da Shell, quando foi pensada para as exigências particulares das plantas de energia e refinarias de petróleo, a tecnologia MPC foi até aplicações envolvendo produção de químicos, processamento de alimentos, aeroespacial (QIN; BAGWELL, 2003). O MPC é considerado uma das técnicas de controle avançado mais bem sucedidas na indútria de processos, é capaz de prover estabilidade, robustez e satisfação de restrições para sistemas lineares e não-lineares (RAWLINGS; MAYNE, 2009) (D'JORGE; FERRAMOSCA; GONZALEZ, 2017).

As aplicações dos MPC's atuais extendem-se para solucionar problemas de otimização submetidos à restrições nas áreas de manufatura avançada, energia, ambiente, aeroespacial, medicina, etc. Verifica-se que existem alguns desafios na manutenção dos controladores preditivos baseados em modelo como parte fundamental do controle de processos industriais de larga escala, como a janela de tempo disponível para o cálculo da ação de controle e a estabilidade do processo (XI; LI; LIN, 2013).

\footnotetext{
* Agradecimentos à CAPES pelo fomento durante a pesquisa.
}

Apesar da popularidade dos controladores preditivos para alguns tipos de malhas de controle, em especial àquelas com restrições e muitas variáveis, existe uma limitação que está ligada a "heterogeneidade"dos controladores preditivos. Uma vez que são capazes de lidar com tantas nuances dos processos, estes devem ser lentos para que exista tempo suficiente para o processamento das ações de controle.

Neste trabalho, os autores propõem uma técnica de obtenção de controlador preditivo com restrições aplicadas as características de robustez do controlador. Estas restrições aplicadas ao diagrama de Nyquist do sistema. Um ambiente didático é utilizado para validar a técnica. Além disso, a utilização de algoritmo genético se faz necessária para resolver um problema de otimização.

A disposição destes aspectos neste trabalho é tal que na seção 2 são dados alguns detalhes a cerca do controle preditivo em espaço de estados, seguindo para a seção 3 onde a técnica é proposta. Na seção 4 é apresentada a análise experimental e por fim, comentários buscando a convergência da contribuição efetiva do trabalho podem ser vistos na seção 5 .

\section{CONTROLE PREDITIVO POR ESPAÇO DE ESTADOS}

O controle preditivo aqui apresentado caracteriza-se como um controlador construído pensando-se na representação em variáveis de estado, que possui estrutura adequada 
para tratamento de sistemas multivariáveis, podendo até mesmo ser estendido para sistemas não-lineares, mas que pode ser passível da implementação de um observador. Num primeiro momento será visto o caso para sistemas SISO (single-input, single-output) e extendidos posteriormente para o caso MIMO (multi-input, multi-output)

Assume-se que o modelo em espaço de estados da planta está bem definido e que um integrador fora inserido. Apesar da noção de um controle preditivo baseado em espaço de estados ter sido baseada nos estudos desenvolvidos recentemente (WANG, 2009), fato é que outros trabalhos anteriores já retratavam essa abordagem (RICKER, 1991). Seja a dinâmica de uma planta dada por (1) para o momento $k$ :

$$
\begin{aligned}
x(k+1) & =A x(k)+B u(k) \\
y(k) & =C x(k)
\end{aligned}
$$

Onde,

- $u$ é variável manipulada ou entrada do processo;

- y é a variável de saída do processo;

- $x(k)$ é o vetor de estados da planta para o instante de tempo $k$ e é assumido como de dimensão $n_{1}$;

- $A, B$ e $C$ são as matrizes que parametrizam o modelo, onde o termo direto $D$ que atuaria sobre a entrada na composição de $y(k)$ foi negligenciado.

\subsection{Ação Integradora}

O próximo passo é a inserção de uma ação integradora no processo, uma vez que estes típicamente conseguem levar sistemas para erro de regime zero. Para tanto, uma análise incremental é sugerida. Tomando o momento $k-1$, são obtidas as equações (3) e (4):

$$
\begin{aligned}
x(k) & =A x(k-1)+B u(k-1) \\
y(k-1) & =C x(k-1)
\end{aligned}
$$

Subtraindo as equações (1) e (3), e considerando que $\Delta u(k)=u(k)-u(k-1)$ e $\Delta x(k+1)=x(k+1)-x(k)$, obtendo-se a $(5)$ :

$$
\Delta x(k+1)=A \Delta x(k)+B \Delta u(k)
$$

Adicionando-se a saída de modo a embarcar a ação integradora, seja agora $x(k)=\left[\Delta x(k)^{T} y(k)\right]^{T}$, tem-se:

$$
\begin{aligned}
y(k+1)-y(k) & =C(x(k+1)-x(k))=C \Delta x(k+1) \\
& =C A \Delta x(k)+C B \Delta u(k)
\end{aligned}
$$

Unindo as equações (5) e (6) e utilizando o fato de que $o_{n_{1}}=\overbrace{\left[\begin{array}{llll}0 & 0 & \ldots & 0\end{array}\right]}^{n_{1}}$, obtemos:

$$
\begin{aligned}
\overbrace{\left[\begin{array}{c}
\Delta x(k+1) \\
y(k+1)
\end{array}\right]}^{x(k+1)}=\overbrace{\left[\begin{array}{cc}
A & o_{n_{1}}^{T} \\
C A & 1
\end{array}\right]}^{A} \overbrace{\left[\begin{array}{c}
\Delta x(k) \\
y(k)
\end{array}\right]}^{x(k)}+\overbrace{\left[\begin{array}{c}
B \\
C B
\end{array}\right]}^{B} \Delta u(k) \\
y(k)=\overbrace{\left[\begin{array}{ll}
C & 1
\end{array}\right]}^{B}\left[\begin{array}{c}
\Delta x(k) \\
y(k)
\end{array}\right]
\end{aligned}
$$

Para que a predição dos estados e das saídas seja efetuada de forma satisfatória, o modelo deve representar efetivamente processo. Assume-se que:

- $A, B$ e $C$ representam o problema;

- $k_{i}$ indica o instante atual;

- $N_{p}$ é a janela de otimização ou horizonte de predição;
- $N_{c}$ é horizonte de controle;

A trajetória futura de controle é denotada por:

$$
\Delta u\left(k_{i}\right), \Delta u\left(k_{i}+1\right), \ldots, \Delta u\left(k_{i}+N_{c}-1\right),
$$

$\mathrm{E}$ os estados futuros preditos são denotadas por:

$$
x\left(k_{i}+1 \mid k_{i}\right), x\left(k_{i}+2 \mid k_{i}\right), \ldots, x\left(k_{i}+N_{p} \mid k_{i}\right)
$$

A leitura destes termos é o estado predito $x\left(k_{i}+m\right)$ dado que são conhecidos os termos até $k_{i}$, onde $m$ é qualquer valor entre 1 e $N_{p}$. Assim, os estados preditos serão:

$$
\begin{aligned}
x\left(k_{i}+1 \mid k_{i}\right) & =A x\left(k_{i}\right)+B \Delta u\left(k_{i}\right) \\
x\left(k_{i}+2 \mid k_{i}\right) & =A x\left(k_{i}+1 \mid k_{i}\right)+B \Delta u\left(k_{i}+1\right) \\
& =A^{2} x\left(k_{i}\right)+A B \Delta u\left(k_{i}\right)+B \Delta u\left(k_{i}+1\right) \\
\vdots & \\
x\left(k_{i}+N_{p} \mid k_{i}\right) & =A^{N_{p}} x\left(k_{i}\right)+A^{N_{p}-1} B \Delta u\left(k_{i}\right)+ \\
& +A^{N_{p}-2} B \Delta u\left(k_{i}+1\right)+\ldots+ \\
& +A^{N_{p}-N_{c}} B \Delta u\left(k_{i}+N_{c}-1\right)
\end{aligned}
$$

Uma vez que os estados preditos estão disponíveis, é possível computar as saídas preditas:

$$
\begin{aligned}
y\left(k_{i}+1 \mid k_{i}\right) & =C A x\left(k_{i}\right)+C B \Delta u\left(k_{i}\right) \\
y\left(k_{i}+2 \mid k_{i}\right) & =C A^{2} x\left(k_{i}\right)+C A B \Delta u\left(k_{i}\right)+C B \Delta u\left(k_{i}+1\right) \\
y\left(k_{i}+3 \mid k_{i}\right) & =C A^{3} x\left(k_{i}\right)+C A^{2} B \Delta u\left(k_{i}\right)+C A B \Delta u\left(k_{i}+1\right) \\
& +C B \Delta u\left(k_{i}+2\right) \\
& \vdots \\
y\left(k_{i}+N_{p} \mid k_{i}\right) & =C A^{N_{p}} x\left(k_{i}\right)+C A^{N_{p}-1} B \Delta u\left(k_{i}\right)+ \\
& +C A^{N_{p}-2} B \Delta u\left(k_{i}+1\right)+ \\
& +\ldots+C A^{N_{p}-N_{c}} B \Delta u\left(k_{i}+N_{c}-1\right)
\end{aligned}
$$

Em ambos os conjuntos de equações listados acima fica claro que os valores preditos dependem unicamente o estado atual do qual se tem informações $\left(x\left(k_{i}\right)\right)$ e da entrada futura $\left(\Delta u\left(k_{i}+j\right)\right.$, com $\left.j=0,1, \ldots, N_{c}-1\right)$, de modo que:

$Y=\left[y\left(k_{i}+1 \mid k_{i}\right) y\left(k_{i}+2 \mid k_{i}\right) y\left(k_{i}+3 \mid k_{i}\right) \ldots y\left(k_{i}+N_{p} \mid k_{i}\right)\right]^{T}$

$\Delta U=\left[\Delta u\left(k_{i}\right) \Delta u\left(k_{i}+1\right) \Delta u\left(k_{i}+2\right) \ldots \Delta u\left(k_{i}+N_{c}-1\right)\right]^{T}$ Nos levando à equação matrícial, tal qual em (8):

$$
Y=F x\left(k_{i}\right)+\Phi \Delta U
$$

Onde,

$$
F=\left[\begin{array}{c}
C A \\
C A^{2} \\
\vdots \\
C A^{N_{p}}
\end{array}\right], \Phi=\left[\begin{array}{ccc}
C B & \ldots & 0 \\
\vdots & \ddots & \vdots \\
C A^{N_{p}-1} B & \ldots & C A^{N_{p}-N_{c}} B
\end{array}\right]
$$

\subsection{Problema de Otimização}

Constrói-se então um problema de otimização, que utilizase das variáveis $Y$ e $\Delta U$ para construir a função objetivo, dada por (9).

$$
J=\left(R_{s}-Y\right)^{T}\left(R_{s}-Y\right)+\Delta U^{T} \bar{R} \Delta U
$$

Em que $R_{s}$ é o vetor referência $\left(r\left(k_{i}\right)\right)$ : 


$$
R_{s}=\overbrace{\left[\begin{array}{llll}
1 & 1 & \ldots & 1
\end{array}\right]^{T}}^{N_{p}} r\left(k_{i}\right)
$$

O primeiro termo da função objetivo (9) está associado a minimização do erro quadrático entre a predição e a referência enquanto o segundo termo está associado à agressividade da saída $\Delta U$. A solução ótima pode ser encontrada substituindo $Y=F x\left(k_{i}\right)+\Phi \Delta U$ na função de custo (9):

$$
\begin{aligned}
J & =\left(R_{s}-F x\left(k_{i}\right)\right)^{T}\left(R_{s}-F x\left(k_{i}\right)\right)- \\
& -2 \Delta U^{T} \Phi^{T}\left(R_{s}-F x\left(k_{i}\right)\right)+\Delta U^{T}\left(\Phi^{T} \Phi+\bar{R}\right) \Delta U
\end{aligned}
$$

Se não existem restrições, um ponto de mínimo pode ser obtido onde a derivada assumir valor zero, tal que:

$$
\frac{\partial J}{\partial \Delta U}=-2 \Phi^{T}\left(R_{s}-F x\left(k_{i}\right)\right)+2\left(\Phi^{T} \Phi+\bar{R}\right) \Delta U
$$

Assumindo que a inversa de $\left(\Phi^{T} \Phi+\bar{R}\right)$ existe:

$$
\Delta U=\left(\Phi^{T} \Phi+\bar{R}\right)^{-1} \Phi^{T}\left(R_{s}-F x\left(k_{i}\right)\right)
$$

Sendo assim (13) a equação de cálculo da ação de controle.

\subsection{Horizonte de Controle Recente}

Apesar de obter-se $N_{c}$ valores, é possível ater-se apenas ao primeiro valor da ação de controle calculada, uma vez que no proximo passo sempre tem-se valores atualizados da saída geral do sistema (BORRELLI ALBERTO BEMPORAD, 2017) (WANG, 2009). Isto pode ser traduzido, reescrevendo a equação (13) de modo a explicitar o único valor de $\Delta U$ a ser utilizado, $\Delta u\left(k_{i}\right)$ :

$$
\begin{aligned}
\Delta u\left(k_{i}\right) & =\left(\Phi^{T} \Phi+\bar{R}\right)^{-1}\left(\Phi^{T} R_{s}-\Phi^{T} F x\left(k_{i}\right)\right) \\
& =K_{y} r\left(k_{i}\right)-K_{x} x\left(k_{i}\right)
\end{aligned}
$$

Onde,

$$
\begin{gathered}
K_{y}=\text { primeiro elemento de }\left(\Phi^{T} \Phi+\bar{R}\right)^{-1} \Phi^{T} R_{s} \\
K_{x}=\text { primeira linha de }\left(\Phi^{T} \Phi+\bar{R}\right)^{-1} \Phi^{T} F
\end{gathered}
$$

\subsection{MPC com Restrições}

O tratamento de restrições muitas vezes é visto com um dos obstáculos para a implementação do MPC. Apesar de complicar consideravelmente o processamento da informação, a naturalidade com a qual o MPC lida com restrições e com múltiplas variáveis atua como um atrativo para aplicações industriais. Quando levam-se em conta as restrições, o problema de programação quadrático assume a forma:

$$
\begin{aligned}
J & =\frac{1}{2} x^{T} E x+x^{T} F \\
M x & \leq \gamma
\end{aligned}
$$

As matrizes $E$ e $F$ assumem formas particulares para os problemas de MPC, associados á dinâmica do sistema e aos valores de referência. $M$ e $\gamma$, por sua vez, inserem as restrições as quais o sistema está submetido.

\section{CONTROLE PREDITIVO ROBUSTO}

Supondo-se que o modelo é tal qual sugerido e seja o modelo contínuo passível de transformação em função de transferência, obtem-se a seguinte função de transferência:

$$
H(s)=C(s I-A)^{-1} B
$$

Sabe-se ainda que através de um modelo contínuo, é possível obter um modelo discreto. Utilizando (17) e considerando um período de amostragem de $h$, obtem-se o modelo discreto:

$$
\begin{aligned}
x(k+1) & =\Psi x(k)+\Gamma u(k) \\
y(k) & =C x(k)
\end{aligned}
$$

No modelo descrito em (19), as matrizes $\Psi$ e $\Gamma$ são descritas como sendo:

$$
\begin{aligned}
& \Psi=e^{A h} \\
& \Gamma=\int_{0}^{h} e^{A s} d s B
\end{aligned}
$$

Em que as matrizes B e A que aparecem em (21) são as matrizes associadas ao modelo contínuo. Sabe-se que em mãos de um modelo discreto em espaço de estados, pode-se obter um controlador preditivo, assim como mostrado no anteriormente. $\mathrm{O}$ primeiro passo é a adição de uma parcela integradora:

$$
\begin{aligned}
\overbrace{\left[\begin{array}{c}
\Delta x(k+1) \\
y(k+1)
\end{array}\right]}^{x(k+1)}=\overbrace{\left[\begin{array}{cc}
\Psi & o_{n_{1}}^{T} \\
\left.\begin{array}{c}
\Psi \\
n_{1}
\end{array}\right] & 1
\end{array}\right]}^{\Psi} \overbrace{\left[\begin{array}{c}
\Delta x(k) \\
y(k)
\end{array}\right]}^{x(k)}+\overbrace{\left[\begin{array}{c}
\Gamma \\
C \Gamma
\end{array}\right]}^{\Gamma} \Delta u(k) \\
y(k)=\overbrace{\left[\begin{array}{ll}
o_{n_{1}} & 1
\end{array}\right]\left[\begin{array}{c}
\Delta x(k) \\
y(k)
\end{array}\right]}^{C}
\end{aligned}
$$

\subsection{Predição de Estados e Saídas}

Em seguida, tendo-se obtido um modelo com a ação integral adicionada, obtem-se as predições dos estados futuros e saídas futuras, como pode-se observar na equação (23):

$$
Y=F x\left(k_{i}\right)+\Phi \Delta U
$$

Sendo $N_{p}$ o horizonte de predição e $N_{c}$ o horizonte de controle. As matrizes $F$ e $\Phi$ são definidas a seguir, tal qual na seção anterior.

Onde,

$F=\left[\begin{array}{c}C \Psi \\ C \Psi^{2} \\ \vdots \\ C \Psi^{N_{p}}\end{array}\right], \Phi=\left[\begin{array}{cccc}C \Gamma & 0 & \ldots & 0 \\ C \Psi \Gamma & C \Gamma & \ldots & 0 \\ \vdots & \vdots & \ddots & \vdots \\ C \Psi^{N_{p}-1} \Gamma & C \Psi^{N_{p}-2} \Gamma & \ldots & C \Psi^{N_{p}-N_{c}} \Gamma\end{array}\right]$

\subsection{Associada as Retrições de Robustez}

Seja o sistema contínuo associado tal qual descrito no início do capítulo, pode-se discretizar o sistema segundo um tempo amostragem $T$ e obter um sistema discreto. Seja este descrito por $H(z)$, podendo então avaliar as partes reais e imaginárias de $H(z)$ de modo que:

- $\Re\left\{\left(e^{\omega_{k}}\right)\right\}$ - parte real da função de transferência na frequência $\omega_{k}$.

- $\Im\left\{\left(e^{\omega_{k}}\right)\right\}$ - parte imaginária da função de transferência na frequência $\omega_{k}$.

O problema de otimização terá então as restrições de robustez aplicadas ao diagrama de Nyquist (KARIMI; 
KUNZE; LONGCHAMP, 2007) (KARIMI; KUNZE; LONGCHAMP, 2006) (KUNZE; KARIMI; LONGCHAMP, 2007):

$$
\begin{gathered}
\left(\cot (\alpha) \Im\left\{\left(e^{\omega_{k}}\right)\right\}-\Re\left\{\left(e^{\omega_{k}}\right)\right\}\right)+\ell \leq 1, \omega_{k}>\omega_{x} \\
\left(\cos (\beta) \Im\left\{\left(e^{\omega_{k}}\right)\right\}+\operatorname{sen}(\beta) \Re\left\{\left(e^{\omega_{k}}\right)\right\}\right)>-1, \omega_{k}>\omega_{x} \\
\left(\cos (\beta) \Im\left\{\left(e^{\omega_{k}}\right)\right\}+\operatorname{sen}(\beta) \Re\left\{\left(e^{\omega_{k}}\right)\right\}\right) \leq-1, \omega_{k} \leq \omega_{x}
\end{gathered}
$$

Nestas restrições, o ângulo $\alpha$, em conjunto com a variável $\ell$ são parâmetros para a construção da reta (KUNZE; KARIMI; LONGCHAMP, 2007))(KARIMI; KUNZE; LONGCHAMP, 2006). O diagrama de Nyquist do sistema, após inserção do controlador deve ficar limitado à reta $d_{1}$ tal qual visto na figura (1):

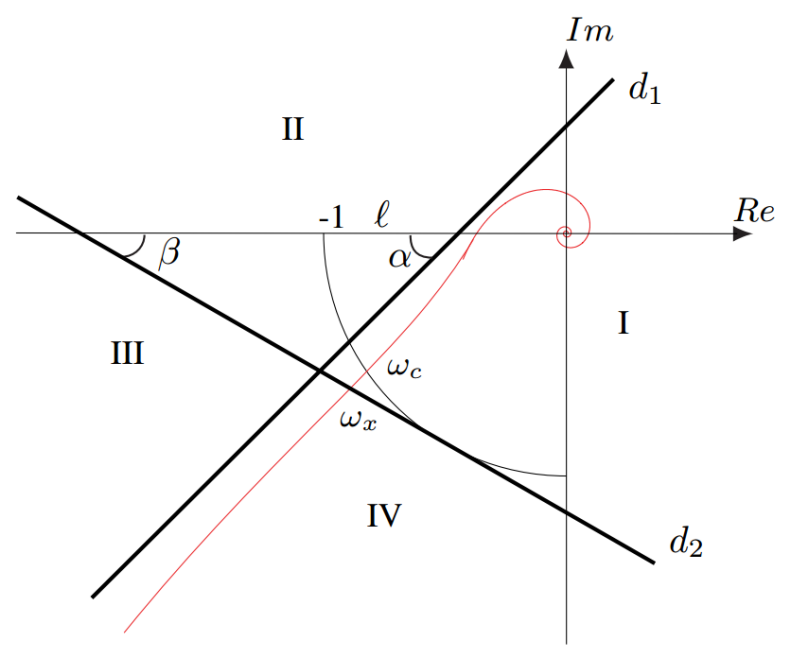

Figura 1. Representação da restrição imposta ao diagrama de Nyquist

A solução ótima pode ser encontrada substituindo $Y=$ $F x\left(k_{i}\right)+\Phi \Delta U$ na função de custo $(9)$ :

$$
\begin{aligned}
J & =\left(R_{s}-F x\left(k_{i}\right)\right)^{T}\left(R_{s}-F x\left(k_{i}\right)\right)-2 \Delta U^{T} \Phi^{T}\left(R_{s}-F x\left(k_{i}\right)\right) \\
& +\Delta U^{T}\left(\Phi^{T} \Phi+\bar{R}\right) \Delta U
\end{aligned}
$$

Seja no entanto $\Delta u\left(k_{i}\right)=K_{y} r\left(k_{i}\right)-K_{m p c} x\left(k_{i}\right)$, onde a referenência está sendo considerada constate.

$$
\Psi^{\dagger}=\left[\begin{array}{c}
\Psi \\
\Psi^{2} \\
\vdots \\
\Psi^{N_{p}}
\end{array}\right], \Gamma^{\dagger}=\left[\begin{array}{cccc}
\Gamma & 0 & \ldots & 0 \\
\Psi \Gamma & \Gamma & \ldots & 0 \\
\vdots & \vdots & \ddots & \vdots \\
\Psi^{N_{p}-1} \Gamma & \Psi^{N_{p}-2} \Gamma & \ldots & \Psi^{N_{p}-N_{c}} \Gamma
\end{array}\right]
$$

De modo que:

$$
X=\Psi^{\dagger} x\left(k_{i}\right)+\Gamma^{\dagger} \Delta U
$$

Assim reescreve-se a entrada $\Delta U$ como sendo:

$$
\begin{aligned}
\Delta U & =K_{r} R_{s}-K_{m p c} X \\
& =K_{r} R_{s}-K_{m p c} \Psi^{\dagger} x\left(k_{i}\right)-K_{m p c} \Gamma^{\dagger} \Delta U \\
\Delta U+K_{m p c} \Gamma^{\dagger} \Delta U & =K_{r} R_{s}-K_{m p c} \Psi^{\dagger} x\left(k_{i}\right) \\
\left(I+K_{m p c} \Gamma^{\dagger}\right) \Delta U & =K_{r} R_{s}-K_{m p c} \Psi^{\dagger} x\left(k_{i}\right) \\
\Delta U & =\left(I+K_{m p c} \Gamma^{\dagger}\right)^{-1} \times \\
& \times\left(K_{r} R_{s}-K_{m p c} \Psi^{\dagger} x\left(k_{i}\right)\right)
\end{aligned}
$$

Dessa forma, recompõe-se a função objetivo, utilizando o valor de $\Delta U$ obtido acima na equação (24), de modo que:

$$
\begin{aligned}
J & =\left(R_{s}-F x\left(k_{i}\right)\right)^{T}\left(R_{s}-F x\left(k_{i}\right)\right) \\
& -2\left[\left(I+K_{m p c} \Gamma^{\dagger}\right)^{-1}\left(K_{r} R_{s}-K_{m p c} \Psi^{\dagger} x\left(k_{i}\right)\right)\right]^{T} \times \\
& \times \Phi^{T}\left(R_{s}-F x\left(k_{i}\right)\right)+ \\
& +\left[\left(I+K_{m p c} \Gamma^{\dagger}\right)^{-1}\left(K_{r} R_{s}-K_{m p c} \Psi^{\dagger} x\left(k_{i}\right)\right)\right]^{T} \times \\
& \times\left(\Phi^{T} \Phi+\bar{R}\right) \times \\
& \times\left[\left(I+K_{m p c} \Gamma^{\dagger}\right)^{-1}\left(K_{r} R_{s}-K_{m p c} \Psi^{\dagger} x\left(k_{i}\right)\right)\right]
\end{aligned}
$$

Claramente, pode-se observar que a função objetivo descrita em (27) é de alta complexidade de cálculo, nos levando a simplificações. Uma delas, pode decorrer do fato de não utilizar ponderação do valor de $\Delta U$, na função objetivo (24), o que nos leva a:

$$
\begin{aligned}
J & =\left(R_{s}-Y\right)^{T}\left(R_{s}-Y\right) \\
& =\left(R_{s}-F x\left(k_{i}\right)-\Phi \Delta U\right)^{T}\left(R_{s}-F x\left(k_{i}\right)-\Phi \Delta U\right) \\
& =\left(R_{s}-F x\left(k_{i}\right)-\Phi\left(I+K_{m p c} \Gamma^{\dagger}\right)^{-1} \times\right. \\
& \left.\times\left(K_{r} R_{s}-K_{m p c} \Psi^{\dagger} x\left(k_{i}\right)\right)\right)^{T} \times \\
& \times\left(R_{s}-F x\left(k_{i}\right)-\Phi\left(I+K_{m p c} \Gamma^{\dagger}\right)^{-1} \times\right. \\
& \left.\times\left(K_{r} R_{s}-K_{m p c} \Psi^{\dagger} x\left(k_{i}\right)\right)\right)
\end{aligned}
$$

Para resolução deste problema de otimização, faz-se necessário a utilização de métodos menos convencionais, tal qual algoritmo genético ou PSO.

\section{RESULTADOS EXPERIMENTAIS}

Para validação da técnica proposta, um módulo didático (LIMA; BARROS; ACIOLI, 2018b) fora utilizado. A associação ocorre de forma simples, onde observa-se uma comunicação serial entre o computador, MATLAB 2013®e o Arduino UNO. O desenvolvimento de tal plataforma e os experimentos associados pode ser observado em referencias (LIMA; BARROS, 2016)(LIMA; BARROS; ACIOLI, 2018a).

O módulo consiste na utilização da dissipação de calor provocada por dispositivos semicondutores, mais precisamente de Transistores de Efeito de Campo (MOSFET) e da consequente propagação deste calor sobre placas de circuito impresso. Na Figura (2) observa-se a disposição de dois transistores e suas distâncias relativas para dois sensores LM35, cuja função é a de obter dados de temperatura da placa de circuito impresso.

Para o experimento referente à obtenção do modelo de primeira ordem com atraso (FOTD), excitaram-se ambas as entradas - uma por vez. O ensaio discorria durante 


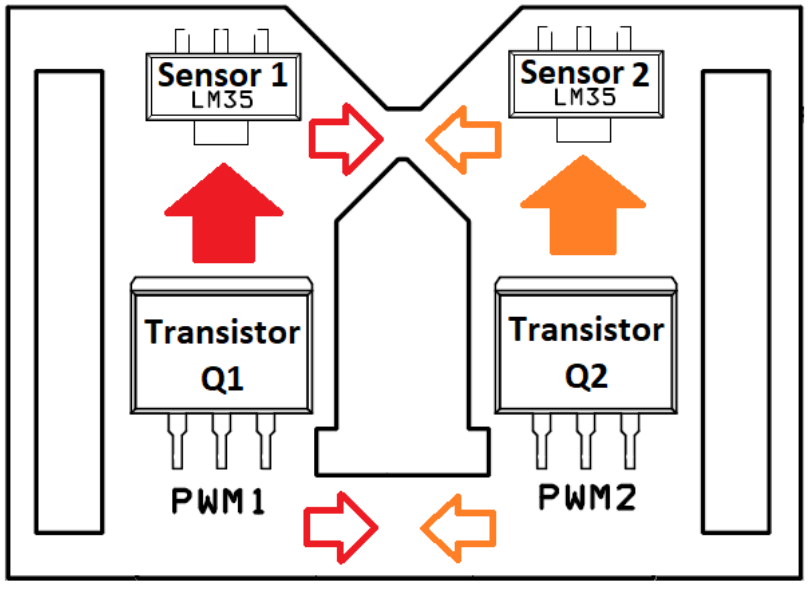

Figura 2. Esquema da disposição dos elementos eletrônicos principais no módulo didático

dois mil segundos (34 minutos) dos quais os primeiros 700 segundos eram destinados a colocar o sistema num ponto de operação desejado. O tempo entre as amostras escolhidos fora de 1 segundo, o que mostrou-se suficiente dado a dinâmica lenta do processo (ASTROM; MURRAY, 2016). A partir daí, eram observados a dinâmica de subida e de descida, dada a ocorrência de degraus sobre o ponto de operação. O sistema obtido pode ser descrito como um sistema MIMO, tal qual descrito na equação (29):

$$
\left[\begin{array}{l}
Y_{1}(s) \\
Y_{2}(s)
\end{array}\right]=\left[\begin{array}{ll}
H_{11}(s) & H_{12}(s) \\
H_{22}(s) & H_{22}(s)
\end{array}\right]\left[\begin{array}{l}
U_{1}(s) \\
U_{2}(s)
\end{array}\right]
$$

As dinâmicas de subida e de descida também apresentam diferenças intrínsecas ao módulo, uma vez que durante o processo de excitação existe uma fonte de energia aplicando calor no módulo e durante o desligamento a superfície do módulo dissipa naturalmente o calor.

No intuito de simplificar os modelos, os autores decidiram pela obtenção de uma única função de transferência que caracterize ambas as dinâmicas, ao custo de perda de ajuste. Num primeiro momento, o interesse é nas funções de transferência diretas, $H_{11}(s)$ e $H_{22}(s)$.

\subsection{Identificação}

Uma alternativa analítica para obtenção de um modelo é a utilização dos dados para a obtenção de um conjunto de parâmetros que descrevam um modelo de primeira ordem com atraso (3 parâmetros) (ACIOLI JUNIOR et al., 2007). Estes parâmetros são vistos evidenciados no modelo abaixo:

$$
G(s)=\frac{Y(s)}{U(s)}=\frac{G_{o}}{\left(T_{1} s+1\right)} e^{-L s}
$$

A técnica baseia-se no método dos mínimos quadrados e o experimento fornece dados do módulo, tal qual pode ser visto na Figura (3). Observe que o sinal inserido fora o mesmo, sendo dividido em um degrau de $40 \%$ que ao um ponto de operação desejado fora adicionado de outro degrau de 10\%. Ao atingir o valor de regime, aplica-se um degrau de $-10 \%$, com relação ao degrau anterior. Na Figura (3), observando-se o comportamento variável da temperatura a partir deste excitação aplicada, em $U_{1}$ e $U_{2}$. Pode-se listar perturbações presentes durante a execução do experimento, como exemplo correntes de ar,
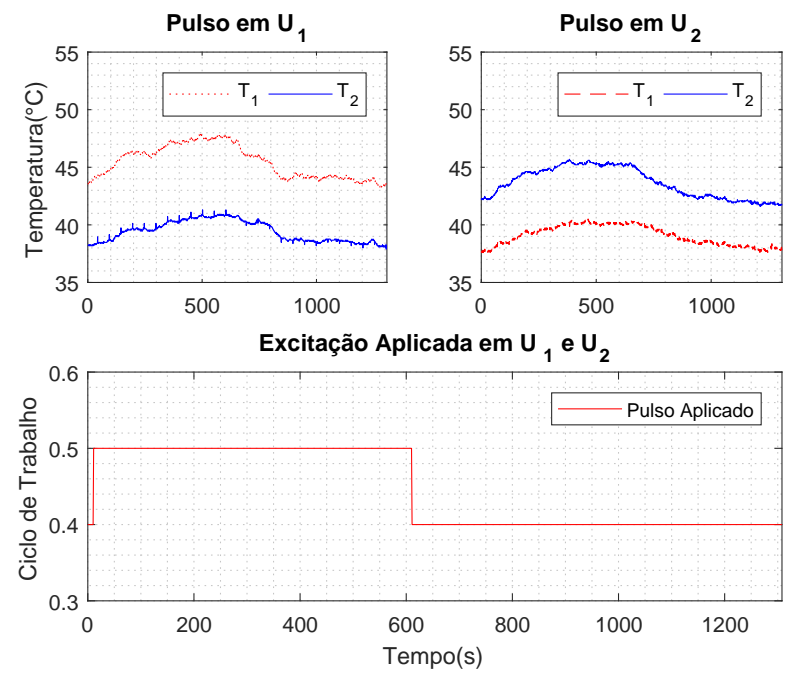

Figura 3. Excitação do sistema em torno do ponto de $50^{\circ}$ Celsius.

aglomerações de pessoas e condicionador de ar. O modelo obtido foi:

$$
H(s)=\left[\begin{array}{cc}
\frac{6,9186}{111,2453 s+1} e^{-2,6 s} & \frac{3,6031}{195,1977 s+1} e^{-2,6 s} \\
\frac{12,0868}{197,0097 s+1} e^{-2,8 s} & \frac{6,6359}{150,3054 s+1} e^{-2,3 s}
\end{array}\right]
$$

Os ajustes foram mantidos acima de 88,46\%, mantendo uma boa fidelidade dos modelos ao sistema real. Taxas melhores não puderam ser obtidas uma vez que ocorre uma divergência entre os modelos que representam a dinâmica de subida e de descida. Utilizando-se da teoria da realização ((LIMA, 2016), obtém-se as seguintes representação em espaço de estados:

- Relativo a $H_{11}$ :

$$
\begin{gathered}
x(k+1)=\left[\begin{array}{cc}
0,9911 & 0,1491 \\
0 & 0
\end{array}\right] x(k)+\left[\begin{array}{c}
0,0998 \\
1
\end{array}\right] u(k-2) \\
y(k)=\left[\begin{array}{ll}
0,2488 & 0
\end{array}\right] x(k)
\end{gathered}
$$

- Relativo a $\mathrm{H}_{22}$ :

$$
\begin{gathered}
x(k+1)=\left[\begin{array}{cc}
0,9934 & 0,0745 \\
0 & 0
\end{array}\right] x(k)+\left[\begin{array}{c}
0,1746 \\
1
\end{array}\right] u(k-2) \\
y(k)=\left[\begin{array}{ll}
0,1766 & 0
\end{array}\right] x(k)
\end{gathered}
$$

\subsection{Controle Preditivo Robusto}

Aplicando-se o algoritmo desenvolvido às parcelas do sistema associadas de forma direta, isto é $H_{11}$ e $H_{22}$, obtémse os controladores. Em ambos os casos, 300 passos temporais foram analisados, cada um consistindo de 3000 amostras e 50 avaliações extras para as melhores soluções. Para $H_{11}$, o vetor associado ao controlador fora:

$$
K_{11}=[0,75351,31200,75356,7116]
$$

Para este caso específico, as variáveis selecionadas foram $\alpha=45^{\circ}, \ell=0,5$, sendo portanto uma restrição leve. Por sua vez, o resultado obtido fora satisfatório, já que os sistema mostrou-se robusto no sentindo de anular a 
perturbação em curta faixa de tempo - com relação à constante de tempo. Além disso, apesar de um impulso ter sido registrado, a saída do sistema alcança apenas um overshoot de $9,5 \%$ do valor de referência, sendo este um bom resultado. Para $H_{22}$, o vetor de controle associado é $K_{22}$ :

$$
K_{22}=[0,78141,22470,78141,4745]
$$

Para este caso específico, as variáveis selecionadas foram $\alpha=60^{\circ}, \ell=0.7$, sendo portanto uma restrição um pouco mais forte, uma vez que as margens de ganho e de fase exigidas é maior. Por sua vez, o resultado obtido fora também satisfatório, igualmente robusto no sentindo de anular a perturbação em curta faixa de tempo - com relação à constante de tempo. Além disso, apesar de um impulso ter sido registrado, a saída do sistema alcança apenas um overshoot de 8,0\% do valor de referência.

Observando-se ambos os desempenhos na figura (4): Além

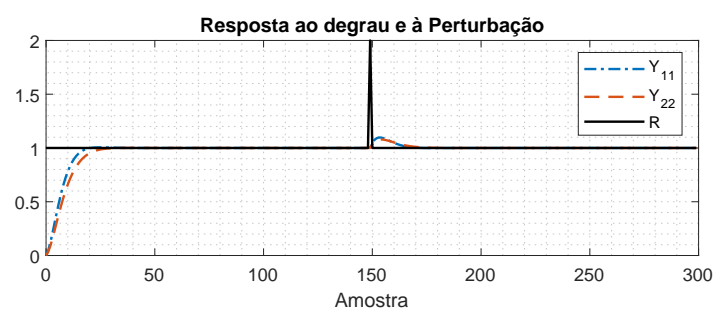

Figura 4. Resposta - Degrau e Perturbação

da resposta no tempo, nota-se a resposta associada as limitações impostas ao diagrama de Nyquist, como mostrado na figura (5):
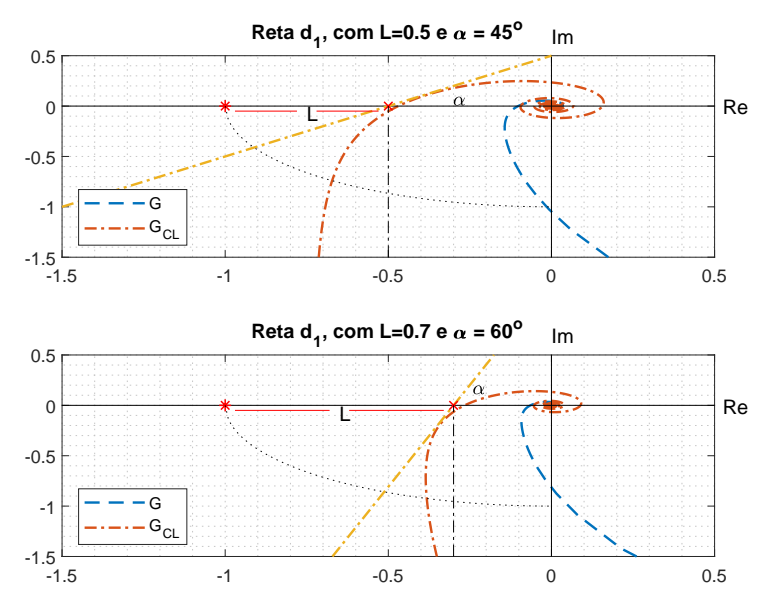

Figura 5. Diagrama de Nyquist para os antes e depois da aplicação do controlador

\section{CONCLUSÕES}

Fato é que a técnica mostrou-se eficaz em prover um controlador preditivo que mantém-se dentro das restrições impostas. Além disso, a resposta no domínio do tempo proporcionou erro de regime zero, o que era esperado dada a presença do integrador.

Um dos problemas que podem ser listados é a alta carga computacional necessária para poder resolver o problema de otimização que é não linear. Essa problemática fora resolvida aqui utilizando o algoritmo genético utilizando uma população gerada no entorno da solução ótima sem restrições. O encurtamento do tempo para o processamento destas soluções e a abertura para soluções subótimas é algo a ser investigado em momentos futuros.

\section{AGRADECIMENTOS}

Agradecimentos à CAPES e à Universidade Federal de Campina Grande.

\section{REFERÊNCIAS}

ACIOLI JUNIOR, G. et al. Identification of firstorder plus dead-time continuous-time models using timefrequency information. VIII SBAI (Simpósio Brasileiro de Automação Inteligente), 2007.

ASTROM, K. J.; MURRAY, R. M. Feedback Systems: An Introduction for Scientists and Engineers. Princeton, NJ, USA: Princeton University Press, 2016.

BORRELLI ALBERTO BEMPORAD, M. M. F. Predictive Control for Linear and Hybrid Systems. [S.1.]: Cambridge University Press, 2017.

D'JORGE, A.; FERRAMOSCA, A.; GONZALEZ, A. H. A robust gradient-based $\mathrm{mpc}$ for integrating real time optimizer (rto) with control. Jornal of Process Control, v. 54 , p. $65-80,2017$.

KARIMI, A.; KUNZE, M.; LONGCHAMP, R. Robust pid controller design by linear programming. In: 2006 American Control Conference. [S.l.: s.n.], 2006. p. 6 pp.--. ISSN 0743-1619.

KARIMI, A.; KUNZE, M.; LONGCHAMP, R. Robust controller design by linear programming with application to a double-axis positioning system. Control Engineering Practice, v. 15, n. 2, p. 197 - 208, 2007. ISSN 0967-0661. KUNZE, M.; KARIMI, A.; LONGCHAMP, R. Gainscheduled controller design by linear programming. In: Control Conference (ECC), 2007 European. [S.l.: s.n.], 2007. p. 5432-5438.

LIMA, A. B. de; BARROS, P. R. Aplicação de otimização linear em projeto de controlador pid. XIII CONGRESSO DE INICIAÇÃO CIENTÍFICA DA UNIVERSIDADE FEDERAL DE CAMPINA GRANDE, 2016.

LIMA, A. B. de; BARROS, P. R.; ACIOLI, G. Módulo didático para ensino de teoria de controle. Congresso Brasileiro de Automática, 2018. ISSN 2525-8311.

LIMA, A. B. de; BARROS, P. R.; ACIOLI, G. Plataforma didática para ensino de identificação e sintonia de controladores pi. Congresso Brasileiro de Automática, 2018. ISSN 2525-8311.

LIMA, R. B. C. de. Metodologia para identificação de sistemas em espaço de estados por meio de excitações pulsadas. [S.1.]: Tese de Doutorado, Universidade Federal de Campina Grande, 2016.

QIN, S. J.; BAGWELL, T. A. A survey of industrial model predictive control technology. Control Engineering Practice, v. 11, n. 7, p. 733-764, 2003.

RAWLINGS, J.; MAYNE, D. Model Predictive Control: Theory and Design. [S.l.]: Nob-Hill Publishing, 2009.

RICKER, N. Model predictive control: State of the art, chemical process control. International Conference on Chemical Process Control, v. 4, n. 4, p. 271-296, 1991.

WANG, L. Model Predictive Control System Design and Implementation Using MATLAB. [S.l.]: Springer, 2009.

XI, Y.-G.; LI, D.-W.; LIN, S. Model predictive control status and challenges. Acta Automatica Sinica, ScienceDirect, v. 39, n. 3, p. 222-236, 2013. 